\title{
The Superluminal Neutrinos from Deformed Lorentz Invariance
}

\author{
Yunjie Huo, ${ }^{1}$ Tianjun Li, ${ }^{1,2}$ Yi Liao, ${ }^{3,4}$ Dimitri V. Nanopoulos, ${ }^{2,5,6}$ Yonghui Qi ${ }^{1}$ and Fei Wang ${ }^{7}$ \\ 1 State Key Laboratory of Theoretical Physics, Institute of Theoretical Physics, \\ Chinese Academy of Sciences, Beijing 100190, P. R. China \\ ${ }^{2}$ George P. and Cynthia W. Mitchell Institute for Fundamental Physics and Astronomy, \\ Texas A\&M University, College Station, TX 77843, USA \\ ${ }^{3}$ Center for High Energy Physics, Peking University, Beijing 100871, P. R. China \\ ${ }^{4}$ School of Physics, Nankai University, Tianjin 300071, P. R. China \\ ${ }^{5}$ Astroparticle Physics Group, Houston Advanced Research Center (HARC), Mitchell Campus, Woodlands, TX 77381, USA \\ ${ }^{6}$ Academy of Athens, Division of Natural Sciences, \\ 28 Panepistimiou Avenue, Athens 10679, Greece \\ ${ }^{7}$ Department of Physics, Zhengzhou University, Zhengzhou, Henan, P. R. China
}

\begin{abstract}
We study two superluminal neutrino scenarios where $\delta v \equiv(v-c) / c$ is a constant. To be consistent with the OPERA, Borexino, and ICARUS experiments and with the SN1987a observations, we assume that $\delta v_{\nu}$ on the Earth is about three order larger than that on the interstellar scale. To explain the theoretical challenges from the Bremsstrahlung effects and pion decays, we consider the deformed Lorentz invariance, and show that the superluminal neutrino dispersion relations can be realized properly while the modifications to the dispersion relations of the other Standard Model particles can be negligible. In addition, we propose the deformed energy and momentum conservation laws for a generic physical process. In Scenario I the momentum conservation law is preserved while the energy conservation law is deformed. In Scenario II the energy conservation law is preserved while the momentum conservation law is deformed. We present the energy and momentum conservation laws in terms of neutrino momentum in Scenario I and in terms of neutrino energy in Scenario II. In such formats, the energy and momentum conservation laws are exactly the same as those in the traditional quantum field theory with Lorentz symmetry. Thus, all the above theoretical challenges can be automatically solved. We show explicitly that the Bremsstrahlung processes are forbidden and there is no problem for pion decays.

PACS numbers: $11.10 . \mathrm{Kk}, 11.25 . \mathrm{Mj}, 11.25 .-\mathrm{w}, 12.60 . \mathrm{Jv}$
\end{abstract}

Introduction - Recently, the OPERA, Borexino, and ICARUS experiments have measured the muon neutrino $\left(\nu_{\mu}\right)$ velocities with high accuracy through the measurement of the flight time and the distance $(730 \mathrm{~km})$ between the source of the CNGS neutrino beam at CERN (CERN Neutrino beam to Gran Sasso) and their detectors at the underground Gran Sasso Laboratory (LNGS) [1 3]. At the OPERA experiment [1], the arrival time of CNGS muon neutrinos with respect to the one for a particle moving at the speed of light in vacuum is

$$
\left.\delta t=(6.5 \pm 7.4 \text { (stat. })_{-8.0}^{+8.3} \text { (sys.) }\right) \mathrm{ns} .
$$

Thus, we obtain a relative difference of the muon neutrino velocity with respect to the speed of light

$$
\begin{aligned}
\delta v_{\nu} & \equiv \frac{v_{\nu}-c}{c} \\
& \left.=(2.7 \pm 3.1 \text { (stat. })_{-3.3}^{+3.4} \text { (sys.) }\right) \times 10^{-6},
\end{aligned}
$$

The dependence of $\delta t$ on the neutrino energy was investigated as well yielding a null effect. At the Borexino experiment [2], we have

$$
\delta t=(0.8 \pm 0.7 \text { (stat.) } \pm 2.9 \text { (sys. })) \mathrm{ns},
$$

which is well consistent with zero. At $90 \%$ Confidence Level (C.L.), we get

$$
\delta v_{\nu}<2.1 \times 10^{-6} .
$$

Morever, at the ICARUS experiment [3], we have

$$
\delta t=(0.18 \pm 0.69 \text { (stat.) } \pm 2.17 \text { (sys.) }) \mathrm{ns},
$$

which is also well consistent with zero. At 95\% C.L., we get

$$
\delta v_{\nu}<1.6 \times 10^{-6} .
$$

To be concrete, we would like to summarize these experimental results: (1) all the central values for $\delta t$ are non-zero and positive [1-3].; (2) $\delta v_{\nu}$ around $10^{-6}$ is still consistent with all the experiments [1-3] $]$; (3) there is no energy denpendence for muon neutrino velocity [1]. Therefore, in this Letter we would like to study the possible superluminal neutrinos with $\delta v_{\nu}$ around $10^{-6}$ which have no energy dependence. From the theoretical point of view, many groups have already studied the possibility of superluminal neutrinos [4 38 ].

The major challenges to the superluminal neutrino results are the following: (1) Bremsstrahlung effects [15]. The superluminal muon neutrinos with $\delta v_{\nu}$ given in Eq. (2) would lose energy rapidly via Cherenkov-like processes on their ways from CERN to LNGS, and the most important process is $\nu_{\mu} \rightarrow \nu_{\mu}+e^{+}+e^{-}$. Thus, the experiment can not observe the muon neutrinos with energy in excess of $12.5 \mathrm{GeV}$ [15]; Considering such effects, the ICARUS experiment has set a tigh limit $\delta v_{\nu}<$ 
$1.25 \times 10^{-8}$ at $90 \%$ C.L. [39]. (2) Pion decays [16, 19, 22]. The superluminal muon neutrinos with $\delta v_{\nu}$ in Eq. (2) can not have energy larger than about $5 \mathrm{GeV}$ from pion decay process, $\pi^{+} \rightarrow \mu^{+} \nu_{\mu}$ and $\mu \rightarrow \nu_{\mu}+e+\bar{\nu}_{e}$ [19]. Several solutions to these challenges have been proposed as well 24, 28, 34 38].

It is well known that the traditional Lorentz invariance can be superseded by two approaches. In the first approach, there exists a preferred frame of reference for Lorentz symmetry breaking [40 42]. In the second approach, the Lorentz invariance is deformed so that the principle of relativity of intertial frames is preserved, while the Lorentz transformations, the energymomentum relations, and the energy-momentum conservation laws are deformed [43 47]. The above theoretical challenges are valid in the first approach. Interestingly, the Bremsstrahlung processes may be forbidden and the pion decays may not be a problem if the Lorentz symmetry is deformed 24]. However, the studied model can not be consistent with the OPERA results obviously since $\delta v_{\nu}$ is proportional to the neutrino energy square [24].

In this paper, we study the constant $\delta v_{\nu}$ around $10^{-6}$ in two scenarios. To be consistent with the OPERA, Borexino, and ICARUS experiments and with the SN1987a observations [48 50], we assume that $\delta v_{\nu}$ is about $10^{-6}$ on the Earth and is smaller than about $2 \times 10^{-9}$ on the interstellar scale, as suggested in the background dependent Lorentz violation proposals [8, 14, 18, 23]. To explain the above theoretical challenges, we consider the deformed Lorentz invariance, and show that the superluminal neutrino dispersion relations can be realized properly while the modifications to the dispersion relations of the other Standard Model (SM) particles can be very tiny and negligible. Moreover, we propose the deformed energy and momentum conservation laws for a generic physical process. In Scenario I the momentum conservation law is preserved while the energy conservation law is deformed. In Scenario II the energy conservation law is preserved while the momentum conservation law is deformed. We present the energy and momentum conservation laws in terms of neutrino momentum in Scenario I and in terms of neutrino energy in Scenario II. In such formats, the energy and momentum conservation laws are exactly the same as those in the traditional quantum field theory with Lorentz symmetry. Thus, all the above theoretical challenges can be automatically solved. To be concrete, we show that the Bremsstrahlung processes are forbidden and the pion decays are not a problem.

The Superluminal Neutrinos from Deformed Lorentz Invariance - Considering the effective field theory or string theory, we can parametrize the generic $\delta v_{\nu}$ for a neutrino as follows

$$
\delta v_{\nu}=-\frac{m_{\nu}^{2}}{2 P_{\nu}^{2}}+\sum_{n \geq 0} a_{n} \frac{P_{\nu}^{n}}{M_{*}^{n}},
$$

where $m_{\nu}$ and $P_{\nu} \equiv\left|\vec{P}_{\nu}\right|$ are respectively the neutrino mass and momentum, $a_{n}$ are the coefficients, and $M_{*}$ is the effective normalization scale. Note that the OPERA results have weak energy dependence, we can only consider the $a_{0}$ term and $a_{1}$ term. The other terms must be very small if they are not vanish. The pure $a_{1}$ term can not be obtained in the Lorentz violation theory with CPT symmetry [40 42]. Interestingly, in the Type IIB string theory, we can obtain this term naturally by calculating the four-point function [23, 51]. Especially, all the theoretical challenges can be solved in such string scenario [28]. Thus, in this Letter we will concentrate on the $a_{0}$ term, i.e., the constant $\delta v_{\nu}$, as suggested by the OPERA experiment.

With the deformed Lorentz invariance, we can parametrize the generic dispersion relation for the SM particles as follows

$$
E_{A}^{2}=\vec{P}_{A}^{2}+m_{A}^{2}+\xi_{P}^{A} \vec{P}_{A}^{2}+\xi_{P E}^{A} P_{A} E_{A}+\xi_{E}^{A} E_{A}^{2},
$$

where $E_{A}, \vec{P}_{A}$ and $m_{A}$ are respectively the energy, momentum and mass for a SM particle $A$, and $\xi_{P}^{A}, \xi_{P E}^{A}$, and $\xi_{E}^{A}$ are coefficients. Because the SM particle masses are invariant under the deformed Lorentz transformations, $\xi_{P}^{A}, \xi_{P E}^{A}$, and $\xi_{E}^{A}$ are universal functions of $m_{A}$ for all the SM particles, i.e., $\xi_{P}^{A} \equiv \xi_{P}\left(m_{A}\right), \xi_{P E}^{A} \equiv \xi_{P E}\left(m_{A}\right)$, and $\xi_{E}^{A} \equiv \xi_{E}\left(m_{A}\right)$. From the effective field theory with CPT symmetry, we can obtain the $\xi_{P}^{A} \vec{P}_{A}^{2}$ or $\xi_{E}^{A} E_{A}^{2}$ term independently [7, 40 42]. If the $\xi_{P E}^{A} E_{A} P_{A}$ term exists, it must come from the interference term. After redefining the kinetic terms and mass terms for the SM particles, we can have either the $\xi_{P}^{A} \vec{P}_{A}^{2}$ term or the $\xi_{E}^{A} E_{A}^{2}$ term. Thus, we shall study two scenarios: Scenario I with only the $\xi_{P}^{A} \vec{P}_{\nu}^{2}$ term and Scenario II with only the $\xi_{E}^{A} E_{\nu}^{2}$ term.

First, we consider the Scenario I where $\delta v_{\nu}=\xi_{P}^{\nu} / 2$. For simplicity, we choose

$$
\xi_{P}^{A}=\alpha_{P} \frac{m_{A}^{2} M_{\mathrm{IR}}^{2}}{m_{A}^{4}+M_{\mathrm{IR}}^{4}},
$$

where $\alpha_{P}$ is a coefficient, and $M_{\mathrm{IR}}$ is the infrared (IR) scale and will be assumed to be the cosmological constant scale. Choosing $m_{\nu}=0.05 \mathrm{eV}, \xi_{P}^{\nu}=1.0 \times 10^{-6}$, and $M_{\mathrm{IR}}=2.3 \times 10^{-3} \mathrm{eV}$, we get $\alpha_{P}=4.73 \times 10^{-4}$. Note that the electron mass is $0.511 \mathrm{MeV}$, we have $\xi_{P}^{e}=9.57 \times$ $10^{-21}$. It is easy to show that $\xi_{P}^{A}$ for all the rest SM particles are smaller than $\xi_{P}^{e}$. Thus, the modifications to the dispersion relations of the SM particles except the neutrinos are very tiny and can be neglected.

The neutrino dispersion relation is required to be invariant under the deformed boost generators $N_{i}^{\nu}$

$$
\left[N_{i}^{\nu}, E_{\nu}^{2}-\vec{P}_{\nu}^{2}-\xi_{P}^{\nu} \vec{P}_{\nu}^{2}\right]=0
$$

And the Einstein special relativity should be realized at the $\xi_{P}^{A}=0$ limit. Thus, we obtain

$$
\left[N_{i}^{\nu}, E_{\nu}\right]=\beta_{1}\left(P_{\nu}\right)_{i}, \quad\left[N_{i}^{\nu},\left(P_{\nu}\right)_{j}\right]=\frac{1}{\beta_{1}} E_{\nu} \delta_{i j},
$$


where $\beta_{1}=\sqrt{1+\xi_{P}^{\nu}}$.

Let us consider a generic physical process: the initial states include $n$ neutrinos and $n^{\prime}$ other SM particles, and the final states include $m$ neutrinos and $m^{\prime}$ other SM particles. We obtain the momentum and energy conservation laws which are invariant under the deformed Lorentz symmetry

$$
\begin{aligned}
\sum_{k=1}^{n} \vec{P}_{\nu k}^{i}+\sum_{k=1}^{n^{\prime}} \vec{P}_{k}^{i} & =\sum_{k=1}^{m} \vec{P}_{\nu k}^{f}+\sum_{k=1}^{m^{\prime}} \vec{P}_{k}^{f}, \\
\frac{1}{\beta_{1}} \sum_{k=1}^{n} E_{\nu k}^{i}+\sum_{k=1}^{n^{\prime}} E_{k}^{i} & =\frac{1}{\beta_{1}} \sum_{k=1}^{m} E_{\nu k}^{f}+\sum_{k=1}^{m^{\prime}} E_{k}^{f},
\end{aligned}
$$

where $P_{k}$ and $E_{k}$ are respectively the momentum and energy for the other SM particles, and the upper indices $i$ and $f$ denote the initial state and final state, respectively. Interestingly, the momentum conservation law is preserved as well. Note that the neutrino masses are tiny and can be neglected, we get $E_{\nu}=\beta_{1} P_{\nu}$. Thus, the energy conservation law can be rewritten as follows

$$
\sum_{k=1}^{n} P_{\nu k}^{i}+\sum_{k=1}^{n^{\prime}} E_{k}^{i}=\sum_{k=1}^{m} P_{\nu k}^{f}+\sum_{k=1}^{m^{\prime}} E_{k}^{f} .
$$

Therefore, the momentum conservation law in Eq. (12) and energy conservation law in Eq. (14) are the same as those in the traditional quantume field theory with Lorentz symmetry. And then all the theoretical challenges can be solved naturally.

Second, we consider the Scenario II where $\delta v_{\nu}=\xi_{E}^{\nu} / 2$. Similar to the Scenario I, we choose

$$
\xi_{E}^{A}=\alpha_{E} \frac{m_{A}^{2} M_{\mathrm{IR}}^{2}}{m_{A}^{4}+M_{\mathrm{IR}}^{4}},
$$

where $\alpha_{E}=4.73 \times 10^{-4}$. Thus, the modifications to the dispersion relations of the SM particles except the neutrinos are very tiny and can be neglected.

The neutrino dispersion relation is required to be invariant under the deformed boost generators

$$
\left[N_{i}^{\nu}, E_{\nu}^{2}-\vec{P}_{\nu}^{2}-\xi_{E}^{\nu} E_{\nu}^{2}\right]=0 .
$$

Thus, we obtain

$$
\left[N_{i}^{\nu}, E_{\nu}\right]=\frac{1}{\beta_{2}}\left(P_{\nu}\right)_{i},\left[N_{i}^{\nu},\left(P_{\nu}\right)_{j}\right]=\beta_{2} E_{\nu} \delta_{i j},
$$

where $\beta_{2}=\sqrt{1-\xi_{E}^{\nu}}$.

For the generic physical process given in Scenario I, we obtain the momentum and energy conservation laws which are invariant under the deformed Lorentz symmetry

$$
\begin{aligned}
\frac{1}{\beta_{2}} \sum_{k=1}^{n} \vec{P}_{\nu k}^{i}+\sum_{k=1}^{n^{\prime}} \vec{P}_{k}^{i} & =\frac{1}{\beta_{2}} \sum_{k=1}^{m} \vec{P}_{\nu k}^{f}+\sum_{k=1}^{m^{\prime}} \vec{P}_{k}^{f}, \\
\sum_{k=1}^{n} E_{\nu k}^{i}+\sum_{k=1}^{n^{\prime}} E_{k}^{i} & =\sum_{k=1}^{m} E_{\nu k}^{f}+\sum_{k=1}^{m^{\prime}} E_{k}^{f} .
\end{aligned}
$$

Interestingly, the energy conservation law is preserved as well. Note that the neutrino masses are tiny and can be neglected, we have $P_{\nu}=\beta_{2} E_{\nu}$. Thus, the momentum conservation law can be rewritten as follows

$$
\sum_{k=1}^{n} E_{\nu k}^{i} \vec{r}_{\nu k}^{i}+\sum_{k=1}^{n^{\prime}} \vec{P}_{k}^{i}=\sum_{k=1}^{m} E_{\nu k}^{f} \vec{r}_{\nu k}^{f}+\sum_{k=1}^{m^{\prime}} \vec{P}_{k}^{f},
$$

where

$$
\vec{r}_{\nu k}^{i} \equiv \frac{\vec{P}_{\nu k}^{i}}{P_{\nu k}^{i}}, \quad \vec{r}_{\nu k}^{f} \equiv \frac{\vec{P}_{\nu k}^{f}}{P_{\nu k}^{f}} .
$$

Therefore, the momentum conservation law in Eq. (20) and energy conservation law in Eq. (19) are the same as those in the traditional quantume field theory with Lorentz symmetry. And then all the theoretical challenges can be solved naturally.

Theoretical Challenges - We will consider the theoretical challenges, and prove that the Bremsstrahlung processes [15] are forbidden and there is no problem for pion decays [16, 19, 22].

First, let us consider the Bremsstrahlung effects. As an simple example, we show that the most important process $\nu_{\mu} \rightarrow \nu_{\mu}+e^{+}+e^{-}$is forbidden in Scenario I. From the generic energy-momentum conservation laws in Eqs. (12) and (14), we have

$$
\begin{aligned}
& \vec{P}_{\nu}^{i}=\vec{P}_{\nu k}^{f}+\vec{P}_{e^{+}}^{f}+\vec{P}_{e^{-}}^{f}, \\
& P_{\nu}^{i}=P_{\nu}^{f}+E_{e^{+}}^{f}+E_{e^{-}}^{f} .
\end{aligned}
$$

Let us suppose that this process is not forbidden. As we know, the electron and positron masses are about $0.511 \mathrm{MeV}$, and the neutrino masses are around $0.05 \mathrm{eV}$. From Eq. (22) we obtain

$$
\left(\vec{P}_{\nu}^{i}-\vec{P}_{\nu k}^{f}\right)^{2}=\left(\vec{P}_{e^{+}}^{f}+\vec{P}_{e^{-}}^{f}\right)^{2}<\left(E_{e^{+}}^{f}+E_{e^{-}}^{f}\right)^{2},
$$

where the above inequality is achieved by considering the electron and positron masses. Using Eq. (23), we obtain

$$
\left(\vec{P}_{\nu}^{i}-\vec{P}_{\nu k}^{f}\right)^{2}<\left(P_{\nu}^{i}-P_{\nu}^{f}\right)^{2} .
$$

This inequality can not be satisfied obviously, thus, the process $\nu_{\mu} \rightarrow \nu_{\mu}+e^{+}+e^{-}$is indeed forbidden.

Second, let us consider the pion decays via the process $\pi^{+} \rightarrow \mu^{+}+\nu_{\mu}$. Similar to the Refs. 16, 19, 22], we consider the neutrino dispersion relation in Scenario I. In Refs. 16, 19], using the preferred frame of reference, the authors obtained their results by assuming the following threshold condition

$$
m_{\pi} \geq m_{\mu}+\sqrt{\xi_{P}^{\nu}} P_{\nu}
$$

However, this threshold condition is not valid in the deformed Lorentz invariance. 
In addition, in Ref. [22], the authors obtained their results by assuming the following energy and momentum conservation laws

$$
\vec{P}_{\pi}=\vec{P}_{\mu}+\vec{P}_{\nu}, \quad E_{\pi}=E_{\mu}+\sqrt{1+\xi_{P}^{\nu}} P_{\nu} .
$$

However, such laws are obviously different from the generic energy and momentum conservation laws in Eqs. (12) and (14) for the deformed Lorentz invariance. In particular, the conservation laws in Eqs. (12) and (14) have no $\xi_{P}^{\nu}$ dependence, thus, we do not have the severe difficulties with the kinematics of the pion decays.

Conclusion - We studied two superluminal neutrino scenarios where $\delta v$ is a constant. To be consistent with the OPERA, Borexino, and ICARUS experiments and with the SN1987a observations, we assumed that $\delta v_{\nu}$ on the Earth is much larger than that on the interstellar scale. To explain the theoretical challenges, we considered the deformed Lorentz invariance, and showed that the superluminal neutrino dispersion relations can be realized properly while the modifications to the dispersion relations of the other SM particles can be negligible. In addition, we proposed the deformed energy and momentum conservation laws for a generic physical process. In Scenario I the momentum conservation law is preserved while the energy conservation law is deformed. In Scenario II the energy conservation law is preserved while the momentum conservation law is deformed. We presented the energy and momentum conservation laws in terms of neutrino momentum in Scenario I and in terms of neutrino energy in Scenario II. In such formats, the energy and momentum conservation laws are exactly the same as those in the traditional quantum field theory with Lorentz symmetry. Thus, all the theoretical challenges can be automatically solved. To be concrete, we showed that the Bremsstrahlung processes are forbidden and the pion decays are not a problem.

Acknowledgments - This research was supported in part by the Natural Science Foundation of China under grant numbers 10821504 and 11075194 (YH, TL and YQ), 10975078 and 11025525 (YL), and 11105124 (FW), and by the DOE grant DE-FG03-95-Er-40917 (TL and DVN).

[1] T. Adam et al. [OPERA Collaboration], arXiv:1109.4897 [hep-ex].

[2] P. Alvarez Sanchez et al. [Borexino Collaboration], arXiv:1207.6860 [hep-ex].

[3] M. Antonello, B. Baibussinov, P. Benetti, E. Calligarich, N. Canci, S. Centro, A. Cesana and K. Cieslik et al., arXiv:1208.2629 [hep-ex].

[4] J. R. Ellis, N. Harries, A. Meregaglia, A. Rubbia and A. Sakharov, Phys. Rev. D 78, 033013 (2008) arXiv:0805.0253 [hep-ph]].

[5] G. Cacciapaglia, A. Deandrea and L. Panizzi, JHEP 1111, 137 (2011) arXiv:1109.4980 [hep-ph]].
[6] G. Amelino-Camelia, G. Gubitosi, N. Loret, F. Mercati, G. Rosati and P. Lipari, Int. J. Mod. Phys. D 20, 2623 (2011) arXiv:1109.5172 [hep-ph]].

[7] G. F. Giudice, S. Sibiryakov and A. Strumia, Nucl. Phys. B 861, 1 (2012) arXiv:1109.5682 [hep-ph]].

[8] G. Dvali and A. Vikman, JHEP 1202, 134 (2012) arXiv:1109.5685 [hep-ph]].

[9] R. B. Mann, U. Sarkar, arXiv:1109.5749 [hep-ph].

[10] A. Drago, I. Masina, G. Pagliara and R. Tripiccione, Europhys. Lett. 97, 21002 (2012) arXiv:1109.5917 [hep$\mathrm{ph}]$.

[11] M. Li, T. Wang, arXiv:1109.5924 [hep-ph].

[12] C. Pfeifer, M. N. R. Wohlfarth, arXiv:1109.6005 [gr-qc].

[13] Z. Lingli, B. -Q. Ma, arXiv:1109.6097 [hep-ph].

[14] J. Alexandre, J. Ellis and N. E. Mavromatos, Phys. Lett. B 706, 456 (2012) arXiv:1109.6296 [hep-ph]].

[15] A. G. Cohen and S. L. Glashow, Phys. Rev. Lett. 107, 181803 (2011) arXiv:1109.6562 [hep-ph]].

[16] L. Gonzalez-Mestres, arXiv:1109.6630 [physics.gen-ph].

[17] M. Matone, arXiv:1109.6631 [hep-ph].

[18] E. Ciuffoli, J. Evslin, J. Liu, X. Zhang, arXiv:1109.6641 [hep-ph].

[19] X. -J. Bi, P. -F. Yin, Z. -H. Yu and Q. Yuan, Phys. Rev. Lett. 107, 241802 (2011) arXiv:1109.6667 [hep-ph]].

[20] P. Wang, H. Wu, H. Yang, arXiv:1109.6930 [hep-ph].

[21] P. Wang, H. Wu, H. Yang, arXiv:1110.0449 [hep-ph].

[22] R. Cowsik, S. Nussinov and U. Sarkar, Phys. Rev. Lett. 107, 251801 (2011) arXiv:1110.0241 [hep-ph]].

[23] T. Li, D. V. Nanopoulos, arXiv:1110.0451 [hep-ph].

[24] G. Amelino-Camelia, L. Freidel, J. Kowalski-Glikman and L. Smolin, Mod. Phys. Lett. A 27, 1250063 (2012) arXiv:1110.0521 [hep-ph]].

[25] J. W. Moffat, arXiv:1110.1330 [hep-ph].

[26] A. E. Faraggi, arXiv:1110.1857 [hep-ph].

[27] R. A. Konoplya and A. Zhidenko, Phys. Rev. D 86, 023531 (2012) arXiv:1110.2015 [hep-th]].

[28] T. Li and D. V. Nanopoulos, Eur. Phys. J. C 72, 2044 (2012) arXiv:1110.3451 [hep-ph]].

[29] L. -A. Zhao and X. Zhang, arXiv:1110.6577 [hep-ph].

[30] Z. Chang, X. Li and S. Wang, Mod. Phys. Lett. 27, 1250058 (2012) arXiv:1110.6673 [hep-ph]].

[31] C. A. G. Almeida, M. A. Anacleto, F. A. Brito and E. Passos, Eur. Phys. J. C 72, 1855 (2012) arXiv:1111.0093 [hep-ph]].

[32] M. Matone, arXiv:1111.0270 [hep-ph].

[33] B. Alles, Phys. Rev. D 85, $047501 \quad$ (2012) arXiv:1111.0805 [hep-ph]].

[34] J. Evslin, arXiv:1111.0733 [hep-ph].

[35] Z. Lingli and B. -Q. Ma, arXiv:1111.1574 [hep-ph].

[36] S. Mohanty and S. Rao, arXiv:1111.2725 [hep-ph].

[37] M. Li, D. Liu, J. Meng, T. Wang and L. Zhou, arXiv:1111.3294 [hep-ph].

[38] Y. Ling, arXiv:1111.3716 [hep-ph].

[39] M. Antonello et al. [ICARUS Collaboration], Phys. Lett. B 711, 270 (2012) arXiv:1110.3763 [hep-ex]].

[40] D. Colladay, V. A. Kostelecky, Phys. Rev. D55, 67606774 (1997).

[41] G. Amelino-Camelia, J. R. Ellis, N. E. Mavromatos, D. V. Nanopoulos, S. Sarkar, Nature 393, 763-765 (1998).

[42] S. R. Coleman, S. L. Glashow, Phys. Rev. D59, 116008 (1999).

[43] G. Amelino-Camelia, Int. J. Mod. Phys. D11, 35-60 (2002). 
[44] G. Amelino-Camelia, Phys. Lett. B510, 255-263 (2001).

[45] J. Kowalski-Glikman, Phys. Lett. A286, 391-394 (2001).

[46] J. Magueijo, L. Smolin, Phys. Rev. Lett. 88, 190403 (2002).

[47] Phys. Rev. D67, 044017 (2003).

[48] R. M. Bionta et al. [IMB Collaboration], Phys. Rev. Lett. 58 (1987) 1494.
[49] E. N. Alekseev, L. N. Alekseeva, I. V. Krivosheina and V. I. Volchenko, Phys. Lett. B 205 (1988) 209.

[50] K. Hirata et al. [KAMIOKANDE-II Collaboration], Phys. Rev. Lett. 58 (1987) 1490.

[51] T. Li, N. E. Mavromatos, D. V. Nanopoulos, D. Xie, Phys. Lett. B679, 407-413 (2009). 